\title{
Relationship between Histologic Chorioamnionitis and Early Inflammatory Variables in Blood, Tracheal Aspirates, and Endotracheal Colonization in Preterm Infants
}

\author{
JOZEF DE DOOY, CECILE COLPAERT, ANNEMIE SCHUERWEGH, CHRIS BRIDTS, \\ MARC VAN DER PLANKEN, MARGARETHA IEVEN, LUC DE CLERCK, WIM STEVENS, AND \\ LUDO MAHIEU \\ Departments of Paediatrics, Division of Neonatology [J.d.D, L.M.], Pathology [C.C.], Hematology \\ [M.v.d.P.], Microbiology [M.I.], and Immunology [A.S., C.B., L.d.C., W.S.], University of Antwerp, \\ Faculty of Medicine, Universiteitsplein 1, B-2610 Wilrijk, Belgium.
}

\section{ABSTRACT}

\begin{abstract}
Histologic results of the placenta are usually not available within the first days of life. We identified inflammatory variables in tracheal aspirates and blood that were associated with histologic chorioamnionitis (HC). A derivation cohort consisted of 62 neonates and a validation cohort of 57 neonates with a gestational age $<31$ wk and ventilated on $\mathrm{d} 1$. Tracheal aspirates were taken on $\mathrm{d} 1$ and on $\mathrm{d} 3$, if the patient was still ventilated. $\mathrm{HC}$ was diagnosed by light microscopy. Logistic regression was used to identify independent factors in the derivation cohort associated with $\mathrm{HC}$ at $\mathrm{d} 1,2$, and 3. Model performance was studied using receiver operating characteristic curve analysis. Independent factors associated with $\mathrm{HC}$ were, at d 1, tracheal aspirate IL-8 $\geq 917 \mathrm{pg} / \mathrm{mL}$ (odds ratio, 60.7; 95\% confidence interval, 11-328); at d 2, blood C-reactive protein $\geq 14$ $\mathrm{mg} / \mathrm{L}$ (odds ratio, 9.2; 95\% confidence interval, 2-38), blood white blood cell count $\geq 10,400 / \mathrm{mm}^{3}$ (odds ratio, $7.4 ; 95 \%$ confidence interval, 2-28); and at d 3, blood neutrophil count $\geq 4968 / \mathrm{mm}^{3}$ (odds ratio, 14; 95\% confidence interval, 3-57). The association with $\mathrm{HC}$ was less at $\mathrm{d} 3$ (area under receiver operating characteristic
\end{abstract}

curve, 0.77) when compared with the $\mathrm{d} 1$ model (area under the curve, $0.88 ; p=0.09$ ). The models performed equally well in the validation cohort (goodness-of-fit test, $p>0.05$ ). We conclude that the $\mathrm{d} 1$ and $\mathrm{d} 2$ models can be used as diagnostic factors for HC. Tracheal aspirate IL-8 taken immediately after birth was equally accurate in the diagnosis of $\mathrm{HC}$ as systemic inflammatory response at $d 2$ and better than on d 3. (Pediatr Res 54: 113-119, 2003)

$\quad$ Abbreviations
HC, histologic chorioamnionitis
TA, tracheal aspirate
WBC, white blood cell
CRIB, clinical risk index for babies
TNF- $\alpha$, tumor necrosis factor- $\alpha$
CRP, C-reactive protein
ROC, receiver operating characteristic
AUC, area under the ROC curve
NICU, neonatal intensive care unit

Chorioamnionitis is considered to be one of the main causes of preterm labor and has been associated with an adverse perinatal outcome (e.g. cerebral palsy and chronic lung disease) in preterm infants $(1,2)$. Histologic results of the examination of the placenta are usually not available within the first days of life, and prenatal diagnosis of chorioamnionitis remains difficult. An early diagnosis is difficult and can only be made in $6 \%$ to $24 \%$ of these patients by amniotic fluid culture (3). As a result of the "chronic inflammation" of fetal or maternal membranes, elevated proinflammatory cytokine levels are found in the amniotic fluid and ultimately

May 21, 2002; November 15, 2002.

Correspondence: Jozef de Dooy, MD, University Hospital Antwerp, Department Pediatrics, Division Neonatology, Wilrijkstraat 10, B-2650 Edegem, Belgium; e-mail: jd978722@planetinternet.be

DOI: 10.1203/01.PDR.0000069702.25801.D1 in the fetus. These cytokines are associated with preterm labor and delivery, a fetal inflammatory response, and the associated negative outcomes $(4,5)$.

The objective of the present study was to identify variables in the TA and blood of preterm infants, measured within the first $3 \mathrm{~d}$ of life, that are associated with $\mathrm{HC}$ in the placenta. These factors would allow us to identify infants with the highest risk of suffering from the adverse consequences of chorioamnionitis.

\section{METHODS}

Study population. The study is based on two cohorts of neonates, admitted to the NICU of the University Hospital of Antwerp. A first group of 62 neonates, "the derivation cohort," was enrolled between September 1999 and October 2000. The 
second group, the "validation cohort," also consisting of 57 neonates, was admitted between November 2000 and February 2002. All neonates had a gestational age $<31 \mathrm{wk}$ and required ventilation on $\mathrm{d} 1$. The following criteria for ventilation were applied: in the delivery room, infants were deemed surfactant deficient if, in the opinion of the attending clinician, they had clinical signs of respiratory distress (persistent cyanosis after bag ventilation, grunting, severe subcostal tirage); or at the $\mathrm{NICU}$, when severe respiratory acidosis $\left(\mathrm{pH}<7.25, \mathrm{PCO}_{2}>\right.$ $50 \mathrm{~mm} \mathrm{Hg}$ ) on blood gas analysis was found. Only neonates with an available placenta examination were included [119 of 125 patients (95\%) admitted during study period]. Our study population of ventilated neonates represents $86 \%$ of the total number of neonates with a gestational age $<31 \mathrm{wk}$ admitted to our NICU during the study period.

Demographic and clinical characteristics of the neonates were also registered. These included sex, gestational age, birth weight, mortality, days of supplemental oxygen therapy, ventilation duration, use of high-frequency ventilation, intraventricular hemorrhage diagnosed by cerebral ultrasound, two scores for assessing the severity of illness [score for neonatal acute physiology (6) and clinical risk index for babies (CRIB) score (7)], respiratory distress syndrome according to the radiologic criteria of Edwards et al. (8), and surfactant therapy. Pregnancy-related maternal data concerned maternal hypertension, preterm premature rupture of membranes, cesarean section, and multiple pregnancy. The research ethics committee gave approval for the study, and parental informed consent was obtained before patients were enrolled.

Sampling procedure. TA were obtained on d 1, as soon as possible after birth and always before administration of surfactant, and on $\mathrm{d} 3$ if the patient was still ventilated. The TA were obtained in a standard manner by the same operator (J.D.D.) with assistance of the nursing staff. A closed tracheal suction system (Trach Care, Ballard Medical Products, Draper, UT, U.S.A.) was used, allowing sterile suctioning and avoiding ventilator disconnection. The infant's head was turned to the left, and $1 \mathrm{~mL}$ of sterile $0.9 \%$ saline was instilled down the endotracheal tube. After 5 mechanical breaths or $10 \mathrm{~s}$ of high-frequency ventilation, aspiration of tracheal fluid was performed, positioning the catheter tip about $1 \mathrm{~cm}$ beyond the end of the endotracheal tube. The infant's head was then turned to the midline and to the right, at each position repeating the aspiration without instilling saline. The whole procedure was then repeated. The median volume returned was $1.6 \mathrm{~mL}$, range, $0.8-3.4 \mathrm{~mL}$.

Sample processing. An aliquot of the TA was used for microbiological examination (quantitative bacterial cultures including Ureaplasma spp). The remaining TA sample was centrifuged for $10 \mathrm{~min}$ at $160 \mathrm{~g}$ force at $4^{\circ} \mathrm{C}$, and the supernatant was stored at $-80^{\circ} \mathrm{C}$ for subsequent analysis. The cell pellets were washed in PBS.

TA cell differential counts. Differential counts were determined for each TA specimen on cytospin preparations (63 g force, $5 \mathrm{~min}$ ) after a May-Grünwald-Giemsa staining.

TA cytokine determination. Cytokines were determined using a flow cytometric microsphere-based immunoassay, the cytometric bead array (Becton Dickinson Biosciences, San
Jose, CA, U.S.A.) as described by Cook et al. (9). Briefly, six microsphere populations, dyed with a proprietary dye (emission $>650 \mathrm{~nm}$ ) having distinct fluorescence intensities, were used. These beads were coated with capture antibodies against cytokines and mixed with recombinant standards or TA samples together with the phycoerythrin (PE)-conjugated (emission, $585 \mathrm{~nm}$ ) detection cytokine-antibodies to form sandwich complexes, consisting a cytokine antibody-cytokine-PElabeled cytokine antibody. In TA samples, IL-1 $\beta$, IL-8, IL-6, IL-10, IL-12p70, and TNF- $\alpha$ were measured with the human inflammation cytometric bead array (Becton Dickinson Pharmingen, Erembodegem, Belgium). Instrument setup was performed using CaliBRITE beads and cytometer setup beads (Becton Dickinson Biosciences), according to the manufacturer's instructions. Two thousand events were measured and analyzed. A monomeric microsphere population was gated out on forward and side scatter. Data were analyzed in two-color fluorescence dot plots, representing the different microsphere populations (emission $>650 \mathrm{~nm}$ ) and the cytokine concentration (according to PE-emission at $585 \mathrm{~nm}$ ). Mean fluorescence intensity values were collected. Four-parametric logistic calibration curves were used, and results were expressed as per milliliter. The detection limit range was $2-20 \mathrm{pg} / \mathrm{mL}$.

Inflammatory parameters in blood. Results on CRP (mg/L), WBC count $\left(/ \mathrm{mm}^{3}\right)$, neutrophil count $\left(/ \mathrm{mm}^{3}\right)$, and thrombocyte count $\left(/ \mathrm{mm}^{3}\right)$ were obtained on blood samples collected on $\mathrm{d} 1$, 2 , and 3 .

Histologic examination of placenta. $\mathrm{HC}$ was diagnosed by light microscopy and registered in a standardized protocol (10). $\mathrm{HC}$ is characterized by $>10$ polymorphonuclear granulocytes per high-power field in the subchorion, chorion, or amnion (11). Severity of $\mathrm{HC}$ is described in three stages, characterized by presence of scattered (mild form) or solid collections (severe form) of polymorphonuclear cells in the subchorion, chorion, or amnion. The moderate form is situated between the mild and severe forms. For statistical evaluation, we defined $\mathrm{HC}$ as present (mild, moderate, or severe form) or absent. We checked whether this more simplified definition of $\mathrm{HC}$ could decrease the prediction of the inflammatory response.

Acute umbilical vasculitis, characterized by polymorphonuclear cells in the wall of the umbilical artery or vein, was also registered.

Model development. The prediction model for $\mathrm{HC}$ was based on the data obtained in the derivation cohort with variables registered on $\mathrm{d} 1,2$, and 3 of life. First, to determine the individual relationship between the dependent variable (HC) and the independent variables, Pearson $\chi^{2}$ analysis was used for categorical data and Kruskal-Wallis analysis for nonparametric continuous data. Second, variables associated with $\mathrm{HC}$ up to $p$ values $<0.25$ were selected for further multivariate analysis using the forward stepwise logistic regression method. Correlation with $p$ values $<0.05$ was regarded as significant. Third, these continuous data were dichotomized at the cutoff point of highest sensitivity and specificity to be more operationally easy in clinical practice. This method allowed us to develop a prediction model for $\mathrm{HC}$ on $\mathrm{d} 1,2$, and 3 . The performance of the prediction models was analyzed by logistic regression and calculation of the AUC. Fourth, different com- 
binations of the independent variables identified in these $3 \mathrm{~d}$ were tested to evaluate their possible improvement in diagnostic performance. Finally, we compared this model with the model using the original continuous data to check for oversimplification.

Model validation. The predictive model for $\mathrm{HC}$ was validated in the second cohort of neonates by assessing discrimination and calibration. For assessing discrimination, or the ability of the model to predict correctly patients who have $\mathrm{HC}$ and patients who do not have $\mathrm{HC}$, the ROC curve analysis was applied. The calibration was evaluated using an overall goodness-of-fit test as previously described (12). Data were collected using the program Filemaker Pro 5.0 (Filemaker, Inc, Santa Clara, CA, U.S.A.). Analysis was done with the statistical package Stata 7.0 (Stata Corporation, College Station, TX, U.S.A.).

\section{RESULTS}

Patient characteristics. In the derivation cohort, 34 cases of $\mathrm{HC}$ were identified among 62 preterm babies (prevalence rate of $\mathrm{HC}=55 \%$ ), whereas 26 of 57 cases of $\mathrm{HC}$ were found in the validation cohort (prevalence rate of $\mathrm{HC}=46 \%$ ). The prevalence rates did not differ significantly $(p>0.05)$. Of the 34 cases of $\mathrm{HC}$ in the derivation cohort, 17 had umbilical vasculitis $(50 \%)$, whereas in the validation cohort 19 of 26 cases with $\mathrm{HC}$ had umbilical vasculitis (73\%). Table $1 \mathrm{com}-$ pares the demographic characteristics and maternal morbidity of the derivation cohort with those of the validation cohort. The only significant difference between the two groups is the CRIB score, which is significantly higher in the validation cohort. All the other characteristics are equally distributed between the data sets. In the derivation cohort at $\mathrm{d} 1,81 \%$ of the TA were sterile, whereas on $\mathrm{d} 3,53 \%$ of the patients were still ventilated and $82 \%$ of the TA were sterile. In the validation cohort at $\mathrm{d} 1$, $68 \%$ of the TA were sterile. On d 3, 32 neonates $(56 \%)$ were still ventilated and $78 \%$ of the TA were sterile. Colonyforming units varied from 0 to 100,000 . The frequency distributions did not differ between the two cohorts $(p>0.05)$.

We accepted finding an abnormal diagnostic test in the group without chorioamnionitis of $10 \%$ and expected to find an abnormal laboratory test in at least $50 \%$ in the chorioamnionitis group. With a confidence level of $95 \%$ and an $\alpha$ error of 0.05 with $80 \%$ power, we calculated the sample size for the two groups. If chorioamnionitis was present in $40 \%$ to $60 \%$ of the cases, total sample size varied from 51 to 56 .

Model derivation. The results of the bivariate analysis are shown in Table 2. In the group with HC $(n=34)$, two patients died before $\mathrm{d} 2$, resulting in a group of 32 patients on $\mathrm{d} 2$ and d 3. In the group without HC $(n=28)$, one patient died before d 3, which resulted in a group of 27 patients on d 3. Inflammatory factors retained for further analysis on $\mathrm{d} 1$ are $\mathrm{TA}$ IL-1 $\beta$, TA IL-6, TA IL-8, TA IL-10, TA IL-12p70, TA TNF- $\alpha$, blood CRP, blood WBC count, blood thrombocyte count, blood neutrophil count, tracheal colonization, TA erythrocyte count, and TA leukocyte count; on d 2, blood CRP, blood WBC count, blood thrombocyte count, and blood neutrophil count; and on d 3, blood WBC count, blood thrombocyte
Table 1. Basic descriptive characteristics in the derivation and validation group

\begin{tabular}{|c|c|c|}
\hline Basic characteristics & $\begin{array}{l}\text { Derivation group } \\
\quad(n=62)\end{array}$ & $\begin{array}{l}\text { Validation group } \\
\quad(n=57)\end{array}$ \\
\hline \multicolumn{3}{|l|}{ Neonatal } \\
\hline \multicolumn{3}{|l|}{ Demographic } \\
\hline Gender: male & $48(30)^{*}$ & $47(27)$ \\
\hline $\begin{array}{l}\text { Gestational age } \\
\text { (weeks) }\end{array}$ & $28(27-29.6) \dagger$ & $28(26-29)$ \\
\hline Birth weight (g) & $947(818-1230)$ & $1000(790-1190)$ \\
\hline \multicolumn{3}{|l|}{ Comorbidity } \\
\hline Dead & $19(12)$ & $28(16)$ \\
\hline SNAP score & $13(9-17)$ & $14(10-17)$ \\
\hline CRIB score & $3(2-7)$ & $7(2-11) \dagger$ \\
\hline $\begin{array}{l}\mathrm{O}_{2} \text { dependency at } \\
\text { PNA-28 }\end{array}$ & $54(27 / 50)$ & $63(26 / 41)$ \\
\hline $\begin{array}{l}\mathrm{O}_{2} \text { dependency at } \\
\text { PMA-36 }\end{array}$ & $22(11 / 50)$ & $37(15 / 41)$ \\
\hline $\mathrm{O}_{2}$ days & $18(4-49)$ & $25(4-52)$ \\
\hline HFV & $48(30)$ & $37(21)$ \\
\hline Ventilation days & $3(2-6)$ & $7(1-13)$ \\
\hline Ventilated on day 3 & $56(35)$ & $68(39)$ \\
\hline $\begin{array}{l}\text { Surfactant } \\
\text { administration }\end{array}$ & $61(38)$ & $67(38)$ \\
\hline IVH & $27(17)$ & $39(22)$ \\
\hline RDS & $84(52)$ & $83(47)$ \\
\hline \multicolumn{3}{|l|}{ Maternal } \\
\hline Multiple pregnancy & $34(21)$ & $19(11)$ \\
\hline pPROM & $31(19)$ & $28(16)$ \\
\hline Caesarian section & $60(37)$ & $55(29)$ \\
\hline Maternal hypertension & $31(19)$ & $28(16)$ \\
\hline
\end{tabular}

SNAP, Score for Neonatal Acute Physiology; CRIB, Clinical Risk Index for Babies; HFV, High Frequency Ventilation; IVH, Intra-Ventricular Hemorrhage; RDS, Respiratory Distress Syndrome; pPROM, Preterm Premature Rupture of Membranes; PNA-28, Postnatal age of 28 days; PMA-36, Postmenstrual age of 36 weeks.

* Percentage (number); $\uparrow$ median $(\mathrm{Q} 1-\mathrm{Q} 3) ; \ddagger p<0.05$ (Pearson $\chi^{2}$ for categorical data, Kruskal-Wallis for continuous data).

count, and blood neutrophil count. Multivariate analysis identified the independent predictors of HC. These were on $\mathrm{d} 1$, TA IL-8; on d 2, blood CRP and blood WBC count (no significant interaction); and on d 3, blood neutrophil count. Although on d 1, blood WBC count (AUC, $0.72 \pm 0.07$ ), blood CRP (AUC, $0.65 \pm 0.05$ ), and blood neutrophil count (AUC, $0.64 \pm 0.07$ ) were associated with $\mathrm{HC}$, their individual performance was significantly inferior to the performance of TA IL-8 (AUC, $0.92 \pm 0.04 ; p<0.05$ ). For each variable, a cutoff point was chosen on the basis of the best percentage of subjects correctly classified (Table 3): d 1, TA IL-8 $\geq 917 \mathrm{pg} / \mathrm{mL}$; d 2, blood $\mathrm{CRP} \geq 14 \mathrm{mg} / \mathrm{L}$ and blood WBC count $\geq 10,400 / \mathrm{mm}^{3}$; and $\mathrm{d}$ 3 , blood neutrophil count $\geq 4,968 / \mathrm{mm}^{3}$. As shown in Table 4, the $\mathrm{d} 1$ model has the best predictive performance (AUC, 0.88) and the $\mathrm{d} 3$ model (AUC, 0.77), the worst performance. The difference between the predictive performance of the $\mathrm{d} 1$ and $\mathrm{d}$ 3 models is borderline significant $(p=0.09)$, whereas the $\mathrm{d} 1$ and d 2 models perform equally well $(p=0.42)$. Although specificity and positive-predictive value of the $\mathrm{d} 3$ model are comparable to the other models, the sensitivity, negativepredictive value, and the fraction of patients correctly classified are inferior. A model composed of the four independent factors found on the first $3 \mathrm{~d}$ did not improve the performance. 


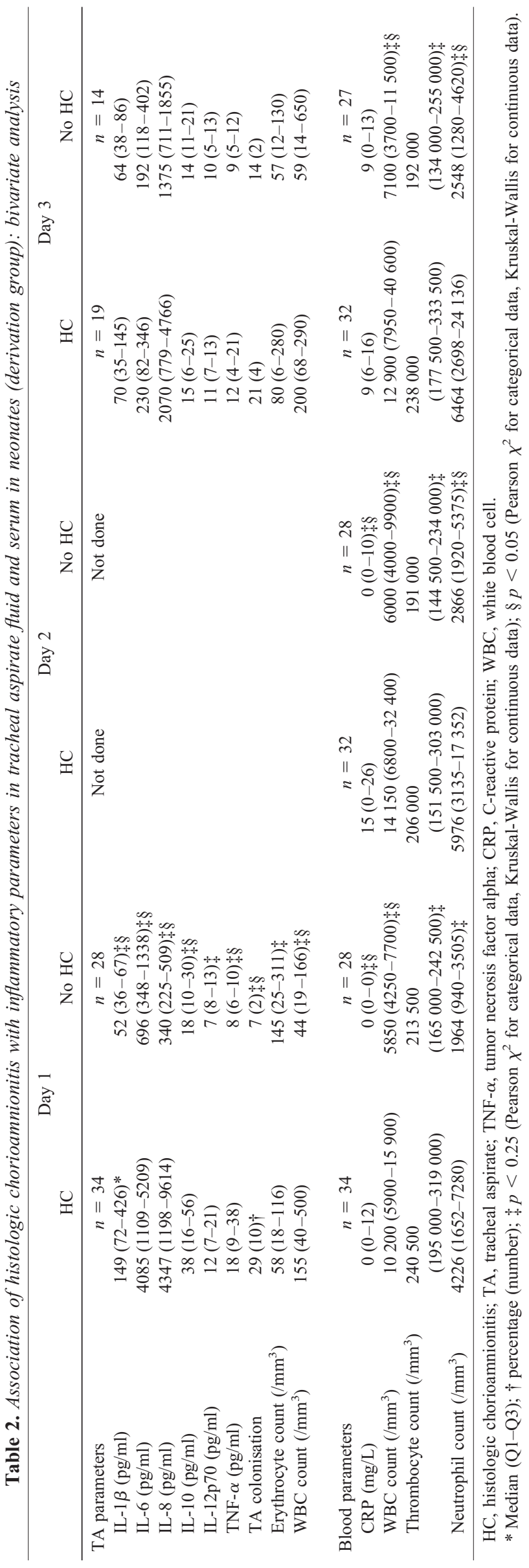

The models based on the original continuous numerical variables did not improve the accuracy of the prediction of $\mathrm{HC}$ compared with the models using the dichotomized data (AUC ROC curves: $\mathrm{d} 1=0.92 \pm 0.04$ versus $0.88 \pm 0.04, p=0.12$; $\mathrm{d} 2=0.84 \pm 0.05$ versus $0.84 \pm 0.07, p=0.88$; and $\mathrm{d} 3=$ $0.78 \pm 0.06$ versus $0.77 \pm 0.05, p=0.76$ ).

We found a significant correlation between IL-8 levels in TA and severity of HC (Spearman's $\rho, 0.73 ; p<0.01$ ). However, the prediction of increased IL-8 level in TA $(\geq 917 \mathrm{pg} / \mathrm{mL})$ was not improved whether chorioamnionitis was defined as present or not present (AUC, $0.88 \pm 0.04$ ) or whether the severity of $\mathrm{HC}$ was used (AUC, $0.86 \pm 0.03 ; p=0.74$ ). Therefore, the use of dichotomized data is operationally easy in clinical practice and correct.

Model validation. Of the 57 patients enrolled in the validation cohort, two patients died before $\mathrm{d} 2$, resulting in a group of 55 patients on $\mathrm{d} 2$ and $\mathrm{d} 3$. The ROC analysis showed that the performance of all the models is equally good in the validation cohort and in the derivation cohort. At a fixed decision criteria of $50 \%$, there was no significant difference in the way the different models correctly classified the patients with $\mathrm{HC}$ in the derivation versus the validation cohort $(p>$ 0.20 ). Because the CRIB score differed significantly between the derivation and validation cohort, we performed an ROC analysis of the different models associated with $\mathrm{HC}$ for different values of the CRIB score. There was not a significant effect on the discriminatory performance of the models (AUC difference between groups was within 0.10 ). The overall goodnessof-fit test for all the models had a $p>0.05$, indicating that the models fit the data and that they are useful in predicting $\mathrm{HC}$.

Finally, to control for selection bias inasmuch as we only included ventilated neonates, we studied the performance of the prediction models of $\mathrm{d} 2$ and $\mathrm{d} 3$ in the group of not ventilated neonates with a gestational age $<31 \mathrm{wk}$ admitted to our NICU during the study period. Because we had no data on cytokine levels in the lung in these 21 not ventilated patients, the performance of the $\mathrm{d} 1$ model could not be determined. No significant differences in the predictive performance of the models were found: $\mathrm{d} 2$ model, derivation cohort (ventilated) AUC ROC $( \pm$ SEM $)=0.84( \pm 0.07)$ versus not ventilated group AUC ROC $( \pm \mathrm{SEM})=0.94( \pm 0.05), p=0.25 ; \mathrm{d} 3$ model, derivation cohort (ventilated) AUC ROC $( \pm$ SEM $)=$ $0.77( \pm 0.05)$ versus not ventilated group AUC ROC ( \pm SEM) $=0.86( \pm 0.08), p=0.34$.

\section{DISCUSSION}

The inflammatory response in the neonate to an infectious challenge such as chorioamnionitis is a complex, dynamic process involving a balance between pro- and antiinflammatory cytokines. We recently described the pathways involved in this process (13). On d 1, we find a significantly higher concentration of TA cytokines IL-1 $\beta$, IL-6, IL-8, IL-10, and TNF- $\alpha$ in patients in the HC group as compared with the control group (Table 2). It is difficult to determine whether cytokines measured in TA soon after birth are of maternal or fetal origin because the TA may be contaminated with amniotic fluid. 
Table 3. Independent factors associated with histologic chorioamnionitis in the derivation cohort (stepwise logistic regression analysis)

\begin{tabular}{lccccc}
\hline & Odds ratio & $95 \%$ CI & Correctly classified $(\%)$ & AUC ROC $( \pm$ SE $)$ & Goodness of fit $(p$-value $)$ \\
\hline Day 1 & 60.7 & $11-328$ & 87 & $0.88( \pm 0.04)$ & 0.99 \\
$\quad$ IL-8 $\geq 917 \mathrm{pg} / \mathrm{mL}$ (TA) & & & & & $0.84( \pm 0.07)$ \\
Day 2 & 9.2 & $2-38$ & 73 & & 0.67 \\
$\quad$ CRP $\geq 14 \mathrm{mg} / \mathrm{L}$ (blood) & 7.4 & $2-28$ & 73 & $0.77( \pm 0.05)$ & 0.98 \\
WBC count $\geq 10400 / \mathrm{mm}^{3}$ (blood) & 14 & $3-57$ & 75 & \\
Day 3 & & & & \\
$\quad$ Neutrophil count $\geq 4968 / \mathrm{mm}^{3}$ (blood) & 14 &
\end{tabular}

95\% CI, 95\% confidence interval; AUC ROC, area under receiver operating characteristic curve of the variable in continuous scale; SE, standard error; TA, tracheal aspirate; CRP, C-reactive protein; WBC, white blood cell.

Table 4. The diagnostic performance of the prediction models of Day 1, Day 2, Day 3, and combination model for histologic chorioamnionitis in the derivation cohort

\begin{tabular}{lcccccc}
\hline & $\begin{array}{c}\text { Sens } \\
(\%)\end{array}$ & $\begin{array}{c}\text { Spec } \\
(\%)\end{array}$ & $\begin{array}{c}\text { PPV } \\
(\%)\end{array}$ & $\begin{array}{c}\text { NPV } \\
(\%)\end{array}$ & $\begin{array}{c}\text { Correctly classified } \\
(\%)\end{array}$ & 87 \\
AUC ROC $( \pm$ SE $)$
\end{tabular}

Sens, sensitivity; Spec, specificity; PPV, positive predictive value; NPV, negative predictive value; AUC ROC, area under receiver operating characteristic curve of the variable in continuous scale; SE, standard error; TA, tracheal aspirate.

$* p<0.05$ when compared to combination model day $1+2+3$.

Many of the cytokines studied have been found in amniotic fluid of women with chorioamnionitis. The inflammatory cytokines IL- $1 \beta$ and TNF- $\alpha$ are substantially increased in the amniotic fluid of women with intrauterine infection (14). IL-6, an important proinflammatory cytokine and mediator of inflammation, can also be found in amniotic fluid, and this might be a reflection of the host response to an intrauterine infection (3). Tsuda et al. (15) found that determining IL-6 levels in amniotic fluid of patients in preterm labor may be of clinical value in establishing the severity of chorioamnionitis and its early diagnosis. Similar to IL-6, IL-8 levels are increased in the amniotic fluid of patients with chorioamnionitis (16).

We have reasons to believe that the inflammatory response that we have found in the TA is to a large extent of fetal origin. Although we did not measure cytokine levels in blood, we find a significant systemic inflammatory response (WBC count, CRP, and neutrophil count) in the neonates of mothers with HC. Some of our findings of cytokine levels measured in TA fluid are in agreement with previous studies on cytokine levels in umbilical cord blood in neonates with HC. Indeed, higher IL- 6 blood levels and IL- $1 \beta$ were found in neonates in whom HC was present (17-19). Elevated cord blood IL-6 concentrations were associated with decreased blood pressure in premature newborns and therefore may predispose such infants to perinatal brain injury (19). It is suggested that preterm newborns with lung inflammation may be unable to activate expression of IL-10, an antiinflammatory cytokine, compared with term newborns with a similar profile of lung inflammation (20). This is not confirmed by our results. We find high levels of IL-10, even significantly higher in the group of premature infants with $\mathrm{HC}$.
On the diagnostic point of view, we find after multivariate analysis that IL-8 in TA fluid remains the only independent and single strongest factor associated with $\mathrm{HC}$ at $\mathrm{d} 1$. To our knowledge, the diagnostic performance of proinflammatory cytokines has not been compared in amniotic fluid of patients with chorioamnionitis. We find that a single measurement of IL-8 in TA taken immediately after birth had a significant better diagnostic value than the measurement of serum inflammatory factors. IL-8 is produced by alveolar macrophages, fibroblasts, type II pneumocytes, and endothelial cells. Hypoxia, hyperoxia, and endotoxins stimulate the production of IL-8. Additional up-regulation of IL-8 is caused by other proinflammatory cytokines such as IL- $1 \beta$. IL- 8 is one of the most important chemotactic factors for neutrophils in the lung (21). Indeed, we find that WBC count is significantly increased in the TA of patients with HC. A previous study found that this factor in TA from preterm infants is significantly correlated with IL-8 levels (22). Similarly, correlations were found between neutrophil counts and IL-8 (23). Our data are supported by a recent study of Schmidt et al. (24) that concluded that HC was associated with an intrauterine cellular inflammatory response and a 7-fold higher expression of IL-8 mRNA in the fetal lung. Finally, the rates of umbilical vasculitis are high in our study population ( $50 \%$ in the derivation group, $73 \%$ in the validation group), also indicating fetal response. Therefore, we believe that the cytokines in the TA are to a large extent of fetal origin.

Our findings suggest that the inflammatory state in the blood as a result of $\mathrm{HC}$ persists during the first days of life, whereas the local inflammatory response in the TA is transient. Indeed, although WBC count, CRP, and neutrophil count in blood are 
associated with $\mathrm{HC}$ on d 1, 2, and 3, cytokine levels and as a consequence WBC count in TA fluid are no longer associated with $\mathrm{HC}$ on d 3. Moreover, we find an increased inflammatory reaction in neonates without $\mathrm{HC}$ on $\mathrm{d} 3$, probably secondary to the effect of ventilation, resulting in comparable cytokine levels on $\mathrm{d}$ 3 in infants with and without HC. Previous studies revealed that elevated CRP in pregnant women with preterm rupture of membranes is a sensitive and specific indicator of chorioamnionitis (25). Moreover, CRP has been found to be elevated in the cord blood of infants with HC (26). We find that the diagnostic value of CRP is obscured by the much more powerful factor IL- 8 at $\mathrm{d}$ 1. A major shortcoming of CRP is its low sensitivity in the acute phase of the disease and the lack of specificity because of the influence of other maternal and neonatal factors, as is seen in neonates with early onset sepsis (27). The sensitivity increases rapidly after birth and is therefore of diagnostic value only at $\mathrm{d} 2$. We suggest that in the absence of data on IL-8 levels in TA at $d$ 1, an accurate clinical diagnosis of $\mathrm{HC}$ can still be made on the basis of the WBC count and the CRP level at $\mathrm{d} 2$, but not at $\mathrm{d} 1$.

Chorioamnionitis is caused by bacteria ascending from the vagina, breaching cervical defenses (mucus plug, and so forth), leading to bacterial colonization of the intrauterine space through intact or ruptured membranes. Organisms vary from normal flora of the vagina to pathogens such as Escherichia coli, Mycoplasma spp, and Ureaplasma spp (1). Tracheal colonization was associated with $\mathrm{HC}$ only during $\mathrm{d} 1$. The pathogens cultured are mostly Gram-positive organisms. We have no data on the relationship between amniotic fluid cultures and pathogens isolated in the TA. It should be noted, however, that amniotic fluid cultures are only helpful in the diagnosis of $\mathrm{HC}$ in $6 \%$ to $24 \%$ (3). The sensitivity of positive TA is also low in our study, on $d 1(29 \%)$ as well as on $d 3(21 \%)$. Moreover, the specificity is as low as $20 \%$ at $\mathrm{d} 3$ but was good at d $1(93 \%)$. In none of the patients with a positive TA on $d 1$ and 3 was the same microorganism found. We suggest that a positive TA on $\mathrm{d} 1$ reflects intrauterine infection and that a positive TA on d 3 is acquired after birth. Owing to the low sensitivity of the TA culture in the diagnosis of $\mathrm{HC}$, we suggest that microbiological investigation of a TA is questionable.

\section{CONCLUSIONS}

We conclude that in ventilated neonates with a gestational age $<31 \mathrm{wk}$, TA IL- $8 \geq 917 \mathrm{pg} / \mathrm{mL}$ on $\mathrm{d} 1$ or blood $\mathrm{CRP} \geq$ $14 \mathrm{mg} / \mathrm{L}$ in combination with WBC count $\geq 10,400 / \mathrm{mm}^{3}$ on $\mathrm{d}$ 2 can be used for the early clinical diagnosis of HC. The neonatal inflammatory response on $\mathrm{d} 3$ is less accurate for the diagnosis of HC. Temporal validation of these diagnostic models in an independent data set shows their accuracy. A shortcoming of our study is that there might be a selection bias as we only included ventilated neonates. We are aware that in other NICUs, the population of ventilated infants could represent a range from almost $100 \%$ to a highly selected group, depending on the criteria used for ventilation. However, analysis of the discriminatory performance of the models of $\mathrm{d} 2$ and d 3 to predict $\mathrm{HC}$ revealed similar results in ventilated and not-ventilated infants. An external validation in an entirely independent sample of patients, ventilated and not ventilated, remains necessary to confirm the strength of the models. Whether these models based on neonatal inflammatory response can also be used for the early prediction of neonatal morbidity and mortality, and then can be used for selection of patients in intervention trials, is a subject for further research.

Acknowledgment. The authors thank Francine Vertessen of the laboratory of hematology for her assistance with the TA cell differential counts.

\section{REFERENCES}

1. Elimian A, Verma U, Beneck D, Cipriano R, Visintainer P, Tejani N 2000 Histologic chorioamnionitis, antenatal steroids, and perinatal outcomes. Obstet Gynecol 96:333336

2. Leviton A, Paneth N, Reuss ML, Susser M, Allred EN, Dammann O, Kuban K, Van Marter LJ, Pagano M, Hegyi T 1999 Maternal infection, fetal inflammatory response, and brain damage in very low birth weight infants. Pediatr Res 46:566-575

3. Romero R, Yoon BH, Mazor M, Gomez R, Diamond MP, Kenney JS, Ramirez M, Fidel PL, Sorokin Y, Cotton D 1993 The diagnostic and prognostic value of amniotic fluid white blood cell count, glucose, interleukin-6, and gram stain in patients with preterm labor and intact membranes. Am J Obstet Gynecol 169:805-816

4. Yoon BH, Romero R, Jun JK, Park KH, Park JD, Ghezzi F, Kim BI 1997 Amniotic fluid cytokines (interleukin-6, tumor necrosis factor- $\alpha$, interleukin- $\beta$, and interleukin-8) and the risk for the development of bronchopulmonary dysplasia. Am J Obstet Gynecol 177:825-830

5. Yoon BH, Romero R, Kim KS, Park JS, Ki SH, Kim BI, Jun JK 1999 A systemic fetal inflammatory response and the development of bronchopulmonary dysplasia. Am J Obstet Gynecol 181:773-779

6. Richardson DK, Gray JE, McCormick MC, Workman K, Goldmann DA 1993 Score for Neonatal Acute Physiology: a physiologic severity index for neonatal intensive care. Pediatrics 91:617-623

7. The International Neonatal Network 1993 The CRIB (clinical risk index for babies) score: a tool for assessing initial neonatal risk and comparing performance of neonatal intensive care units. Lancet 342:193-198

8. Edwards DK, Hilton SV, Merritt TA, Hallman M, Mannino F, Boynton BR 1985 Respiratory distress syndrome treated with human surfactant: radiographic findings. Radiology 157:329-334

9. Cook EB, Stahl JL, Lowe L, Chen R, Morgan E, Wilson J, Varro R, Chan A, Graziano FM, Barney NP 2001 Simultaneous measurement of six cytokines in a single sample of human tears using microparticle-based flow cytometry: allergics $v s$. non-allergics. J Immunol Methods 254:109-118

10. DiSalvo D 1998 The correlation between placental pathology and intraventricular hemorrhage in the preterm infant. The Developmental Epidemiology Network Investigators. Pediatr Res 43:15-19

11. Benirschke K, Kaufmann P 2000 Pathology of the Human Placenta. Springer-Verlag, New York, pp 268-318

12. Mahieu LM, De Muynck AO, De Dooy JJ, Laroche SM, Van Acker KJ 2000 Prediction of nosocomial sepsis in neonates by means of a computer-weighted bedside scoring system (NOSEP score). Crit Care Med 28:2026-2033

13. De Dooy JJ, Mahieu LM, Van Bever HP 2001 The role of inflammation in the development of chronic lung disease in neonates. Eur J Pediatr 160:457-463

14. Saji F, Samejima Y, Kamiura S, Sawai K, Shimoya K, Kimura T 2000 Cytokine production in chorioamnionitis. J Reprod Immunol 47:185-196

15. Tsuda A, Ikegami T, Hirano H, Sanada H, Ogawa M, Sasaki M, Tanaka T 1998 The relationship between amniotic fluid interleukin-6 concentration and histologic evidence of chorioamnionitis. Acta Obstet Gynecol Scand 77:515-520

16. Zhang W, Wang L, Zhao Y, Kang J 2000 Changes in cytokine (IL-8, IL-6 and TNF- $\alpha$ ) levels in the amniotic fluid and maternal serum in patients with premature rupture of the membranes. Zhonghua Yi Xue Za Zhi 63:311-315

17. Buscher U, Chen FC, Pitzen A, Menon R, Vogel M, Obladen M, Dudenhausen JW 2000 Il-1 $\beta$, Il-6, Il-8 and G-CSF in the diagnosis of early-onset neonatal infections J Perinat Med 28:383-388

18. Yoon BH, Romero R, Park JS, Kim M, Oh SY, Kim CJ, Jun JK 2000 The relationship among inflammatory lesions of the umbilical cord (funisitis), umbilical cord plasma interleukin 6 concentration, amniotic fluid infection, and neonatal sepsis. Am J Obstet Gynecol 183:1124-1129

19. Yanowitz TD, Jordan JA, Gilmour CH, Towbin R, Bowen A, Roberts JM, Brozanski BS 2002 Hemodynamic disturbances in premature infants born after chorioamnionitis: association with cord blood cytokine concentrations. Pediatr Res 51:310-316

20. Jones CA, Cayabyab RG, Kwong KY, Stotts C, Wong B, Hamdan H, Minoo P, deLemos RA 1996 Undetectable interleukin (IL)-10 and persistent IL-8 expression early in hyaline membrane disease: a possible developmental basis for the predisposition to chronic lung inflammation in preterm newborns. Pediatr Res 39:966-975

21. Jorens PG, Van Damme J, De Backer W, Bossaert L, De Jongh RF, Herman AG, Rampart M 1992 Interleukin 8 (IL-8) in the bronchoalveolar lavage fluid from patients with the adult respiratory distress syndrome (ARDS) and patients at risk for ARDS. Cytokine 4:592-597 
22. Munshi UK, Niu JO, Siddiq MM, Parton LA 1997 Elevation of interleukin-8 and interleukin-6 precedes the influx of neutrophils in tracheal aspirates from preterm infants who develop bronchopulmonary dysplasia. Pediatr Pulmonol 24:331-336

23. Sheeran P, Jafri H, Carubelli C, Saavedra J, Johnson C, Krisher K, Sanchez PJ, Ramilo O 1999 Elevated cytokine concentrations in the nasopharyngeal and tracheal secretions of children with respiratory syncytial virus disease. Pediatr Infect Dis J 18:115-122

24. Schmidt B, Cao L, Mackensen-Haen S, Kendziorra H, Klingel K, Speer CP 2001 Chorioamnionitis and inflammation of the fetal lung. Am J Obstet Gynecol 185:173-177
25. Beck T, Bahlmann F, Weikel W 1993 Histology of chorioamnionitis: relations to maternal and fetal infection parameters. Z Geburtshilfe Perinatol 197:129-134

26. Miyano A, Miyamichi T, Nakayama M, Kitajima H, Shimizu A 1998 Differences among acute, subacute, and chronic chorioamnionitis based on levels of inflammation-associated proteins in cord blood. Pediatr Dev Pathol 31:513-521

27. Pourcyrous M, Bada HS, Korones SB, Baselski V, Wong SP 1993 Significance of serial C-reactive protein responses in neonatal infection and other disorders. Pediatrics 92:431-435 